\title{
Alice and Bob: Reconciling Formal Models and Implementation (Extended Version)
}

\author{
Omar Almousa ${ }^{1}$, Sebastian Mödersheim ${ }^{1}$, and Luca Viganò ${ }^{2}$ \\ 1 DTU Compute, Lyngby, Denmark \\ 2 Department of Informatics, King's College London, UK
}

\begin{abstract}
This paper defines the "ultimate" formal semantics for Alice and Bob notation, i.e., what actions the honest agents have to perform, in the presence of an arbitrary set of cryptographic operators and their algebraic theory. Despite its generality, this semantics is mathematically simpler than any previous attempt. For practical applicability, we introduce the language SPS and an automatic translation to robust real-world implementations and corresponding formal models, and we prove this translation correct with respect to the semantics.
\end{abstract}

\section{Introduction}

Alice-and-Bob notation is a simple and succinct way to specify security protocols: one only needs to describe what messages are exchanged between the protocol agents in an unattacked protocol run. However, it has turned out to be surprisingly subtle to define a formal semantics for such a notation, i.e., defining an inference system for how agents should compose, decompose and check the messages they send and receive. Such a semantics is necessary in order to automatically generate formal models and implementations from Alice-and-Bob specifications. However, even modeling messages in the free algebra, defining the semantics has proved far from trivial [11-13, 20,22,23]. To make matters worse, many modern protocols rely, for instance, on the Diffie-Hellman key agreement where the algebraic properties of modular exponentiation are necessarily part of the operational semantics, since the key exchange would be non-executable in the free algebra. For practical purposes, one can augment the semantics with support for just this special example like [28], but a general and mathematically succinct and rigorous theory is desirable.

We give in this work a semantics for an arbitrary set of operators and their algebraic properties. Despite this generality, the semantics is a much more succinct and mathematically simple definition than all the previous works (it fits on half a page) because it is based on a few general and uniform principles to define the behavior of the participants. This semantics was inspired by the similar works of $[24,14]$, which we further simplify considerably. Our semantics is also subsuming the previous works in the free algebra and limited algebraic reasoning, as they are instances of our semantics for a particular choice of operators 
and algebraic properties (although this is not easy to show as explained below). We thus see our semantics as one of our main contributions since, from a mathematical point of view, a simple general principle that subsumes the complex definitions of many special cases is the most desirable property of a definition. ${ }^{3}$

This simple mathematical semantics however cannot be directly used as a translator from Alice-and-Bob notation to formal models or implementations since it entails an infinite representation and several of the underlying algebraic problems are in fact not recursive in general. We thus consider a particular set of operators and their algebraic properties that supports a large class of protocols, including modular exponentiation and multiplication. This low-level semantics is much more complex than the mathematical high-level one but it is computable, and we formally prove that the low-level semantics is a correct implementation of the high-level one. We believe it is in fact representative for the theories for which the semantics has been given in past papers, and also clarifies subtle details of the behavior of operators that were left implicit previously. The division into a simple mathematical high-level semantics as a "gold standard" and a low-level "implementable" semantics not only allows for a reasonable correctness criterion of the low-level semantics, but is in our opinion a major advantage over previous works that are a blending between mathematical and technical aspects.

To make our work applicable in practice, we have designed the Security Protocol Specification language SPS as a variant of existing Alice-and-Bob languages that contains many novel features valuable in practice. In particular, our notion of formats allows to integrate the particular way of structuring messages of real-world protocols like TLS, rather than academic toy implementations; at the same time, we can use a sound abstraction of these formats in the formal verification. We have implemented the low-level semantics in a translator that can generate both formal models in the input languages of popular security protocol analysis tools (e.g., Applied $\pi$ calculus in the syntax of ProVerif [10] or ASLAN for AVANTSSAR [5]) and implementations in JavaScript for the execution environment of the FutureID project (www.futureid.eu). We have demonstrated practical feasibility with a number of major and minor case studies, including TLS and the EAC/PACE protocols used in the German eID card.

We proceed as follows: we give the syntax of SPS in Section 2 and an extension of strands in Section 3. We define the semantics of SPS in Section 4 and discuss the connections from SPS to implementations and formal models in Section 5. In Section 6, we discuss related and future work, and conclude the paper.

\section{SPS Syntax}

In this section, we briefly introduce the syntax of SPS, which we will illustrate by referring to the example protocol specification in SPS given in Listing 1.1, in which two agents A and B use a symmetric key $\operatorname{shk}(A, B)$ to establish a fresh Diffie-Hellman key and securely exchange a Payload message.

\footnotetext{
${ }^{3}$ We have learned that from Pierpaolo Degano, who is renowned for his ability to explain complex things in a simple way.
} 


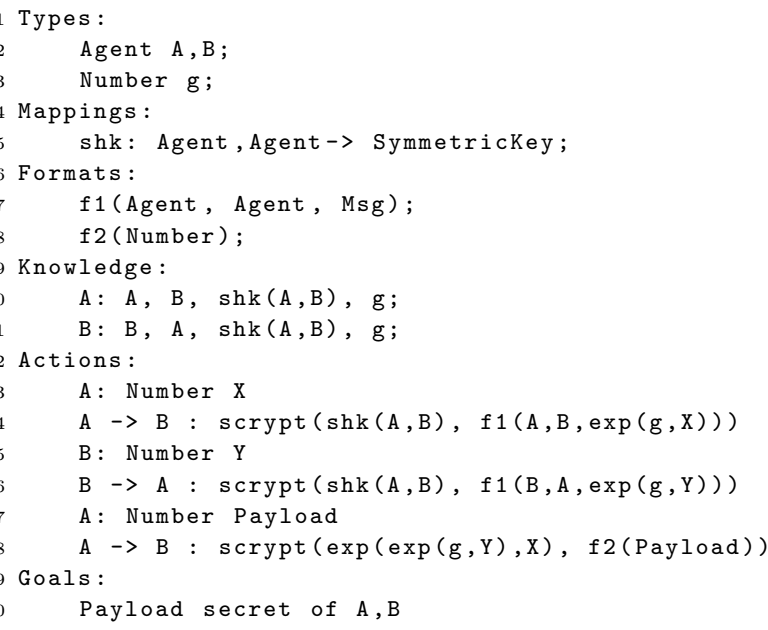

Listing 1.1. Example protocol specification in SPS

We give the syntax of SPS in EBNF, where we set all meta-symbols in blue and write $\mathrm{X} s$ (for a non-terminal symbol $\mathrm{X}$ ) to denote a comma-separated list $\mathrm{X}(, \mathrm{X})^{*}$ of $\mathrm{X}$ elements; CONST and FUNC are alphanumeric strings starting with a lower-case letter (e.g., $g$ and scrypt in the example) and VAR is an alphanumeric string starting with an upper-case letter (e.g., $\mathrm{X}$ in the example).

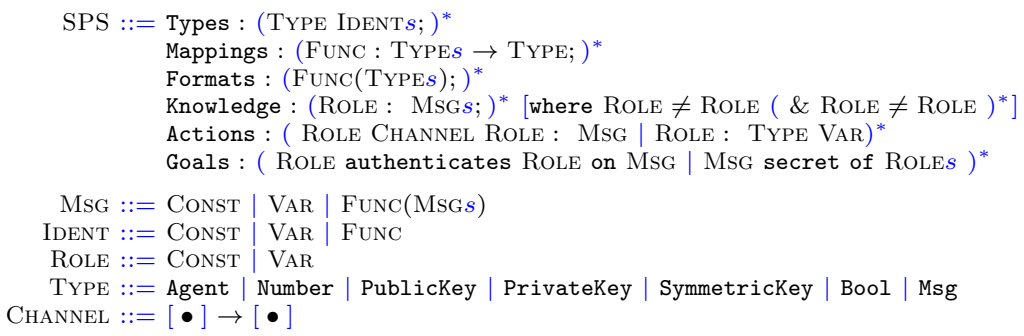

We begin our explanation with the atomic elements: constants (CONST) and variables (VAR). One may think of the variables as parameters of a protocol description that must be instantiated for a concrete execution of the protocol; in our example, the variables A and B shall be instantiated with concrete agent names such as $\mathrm{a}, \mathrm{b}$ or the intruder $\mathrm{p}^{4}$, whereas $\mathrm{X}$ and $\mathrm{Y}$ should be instantiated with random numbers that are freshly chosen by A and B, respectively.

In the Types section, all constants and variables are declared with one of the pre-defined types, where the type Msg subsumes all types. By default, the interpretation of SPS is untyped, i.e., types are used only by the SPS translator to check that the user did not specify any ill-typed terms. The types can however be used to generate a more restrictive typed model and under certain conditions this

\footnotetext{
${ }^{4}$ SPS actually uses the constant $i$, but in this paper we write $\mathrm{p}$ in honor of our "favorite intruder".
} 
restriction is without loss of attacks [4]. The type Agent has a special relevance: constants and variables of this type we call roles, and the symbol ROLE in the above grammar must only be used for identifiers of type Agent. (This is an additional check we cannot directly express in a context-free grammar.)

While the semantics of Alice-and-Bob style languages that we give in the next section is generic for an arbitrary set of function symbols and their algebraic properties, the concrete implementation of SPS is for a set of fixed cryptographic function symbols. These are asymmetric and symmetric encryption (crypt and scrypt), digital signatures (sign), hash and keyed-hash functions (hash and mac), and modular exponentiation (exp) and multiplication (mult). There are of course corresponding operations for decryption and verification, but these are not part of an SPS specification; instead, their use is derived by the SPS translator according to the semantics in the next section.

In the Mappings section, one can specify a special kind of function symbols. These do not represent any actual operation that honest agents or the intruder can perform, but are used to describe pre-existing setup of long-term keys. In our example, the mapping shk assigns to every pair of agents a unique value of type symmetric key; this is the easiest way to define shared keys for agents - including the intruder who will then share $\operatorname{keys} \operatorname{shk}(\mathrm{p}, \mathrm{A})$ and $\operatorname{shk}(\mathrm{A}, \mathrm{p})$ with every other agent $\mathrm{A}$. Public key infrastructures can be modeled in a similar way.

In the Formats section, one can specify a third kind of function symbols called formats. They abstractly represent how the concrete implementation structures the clear-text part of a message, such as XML-tags or explicit message-length fields. A format thus basically represents a concatenation of information, but in contrast to a plain concatenation operator as in other formal languages, the abstract format function symbols allow us later to generate implementations with real-world formats such as TLS. In the example, we have two formats: $f 1$ is used to exchange the Diffie-Hellman half-keys together with the agent names, and $\mathrm{f} 2$ indicates the transmission of the Payload message. For simplicity, we model a payload message using a fresh random number Payload, representing a placeholder for an arbitrary message (depending on the concrete application); alternatively, this could be modeled using a mapping (e.g., payload $(A, B)$ ) that A knows initially and sends to B after the key establishment.

The three kinds of function symbols are thus: the cryptographic function symbols, the mappings and the formats. Except for the mappings, these are all public: all agents, including the intruder, can apply them to messages they know. Additionally, formats are transparent: every agent can extract the fields of a format. We can now build composed messages with these function symbols, where we assume the additional check that all SPS messages are well-typed (and are used with the proper arity). As typing is not essential for this paper, we do not discuss the details of the type expressions.

In the Knowledge section, we specify the initial knowledge of each of the protocol roles. This is essential as it determines how (and if) honest agents can execute the protocol. For instance, if in the example we were to omit the item $\operatorname{shk}(A, B)$ in the knowledge of role $B$, then $B$ could not decrypt the first message 
from A and thus not obtain A's half key. Moreover, in the next step B would be unable to build the response message for A. Also, as we will define below, this specification indirectly determines the initial knowledge of the intruder: if a role is instantiated with $\mathrm{p}$, then the intruder obtains the corresponding knowledge (in our case, all shared $\operatorname{keys} \operatorname{shk}(A, B)$ where $A=p$ or $B=p$ ). We require that all variables in the knowledge section be of type Agent. Finally, one can optionally forbid some instantiations of the roles, e.g., by the side condition $\mathrm{A} \neq \mathrm{p}$ or $\mathrm{A} \neq \mathrm{B}$.

The Actions section is the core of the specification: it specifies the messages that are exchanged between the roles. Additionally, we specify here explicitly when agents freshly create new values. In our example, A first creates the secret exponent $\mathrm{X}$ for the Diffie-Hellman exchange, computes the half-key exp (g, X), inserts it into format $f 1$ and encrypts the message with the shared key shk $(A, B)$. To send this message, A uses the standard insecure channel (denoted with $\rightarrow$ ) on which the intruder can read, intercept, and insert messages arbitrarily. SPS also supports a notion of authentic, confidential, and secure channels as in [24], denoted with $\bullet \rightarrow, \rightarrow \bullet$ and $\bullet \rightarrow \bullet$, respectively. For instance, one may have specified the exchange of the half-keys without the encryption but using authentic channels where the intruder can see messages, but not insert messages except under his real name. This represents the assumption that the messages between $A$ and $B$ cannot be manipulated by an intruder, e.g., in device pairing of mobile devices, when A and B meet physically in a public place. The assumptions are reflected only in the formal model (by restricting the intruder behavior on such channels), while in the implementation it is the duty of the surrounding software module to connect a properly secured channel to the protocol module.

In the final Goals section, we specify the goals the protocol aims to achieve. SPS provides built-in macros for the standard secrecy and authentication goals. We translate these goals to events (that we discuss later) such as $\operatorname{secret}(\mathrm{A}, \mathrm{B}$, Payload), which reflect what is happening in the protocol execution, and attack states are defined as predicates over these events. One can then in general formulate security goals with respect to such events in a protocol-independent way (and depending on what support is offered by the protocol analysis tool considered) rather than referring to the messages of the protocol.

\section{Operational Strands}

As a preparation for defining the SPS semantics, we first clarify the target language, i.e., we define an extension of the popular strands [30] that we call operational strands. Here we give in a glance the five extensions that we make. A concrete example is shown in Fig. 1 and explained below and we give the formal details of operational strands in Appendix A.

First, send and receive steps can be annotated with a channel. Recall that SPS supports standard insecure channels as well as authentic, confidential and secure ones. As far as the SPS semantics is concerned, this is only a label on the channels that are left unchanged. In textual representation, we write send $(c h, t)$ and receive $(c h, t)$ for sending and receiving a message $t$ over a channel $c h$. 
Second, we annotate each strand with the initial knowledge of the role, denoted by a box above the strand (we define knowledge formally in Definition 2). In textual representation, we write the annotation with the knowledge $M$ as $M$ : steps at the beginning of the strand.

Third, recall that the original strand spaces are used to characterize sets of protocol executions and contain only ground terms. In contrast, we use them like a "light-weight" process calculus: terms may contain variables (representing values that are instantiated during the concrete execution) and have the construct fresh $X$ where the variable $X$ will be bound to a fresh value. An important requirement is that operational strands are closed in the following sense: every variable must be bound by first occurring in the initial knowledge, in a fresh operation, in a macro (that we shortly introduce), or in a receive step. A bound variable must not occur subsequently in a fresh operation (i.e., it cannot be "re-bound"). In contrast, a bound variable may occur in a subsequent receive step, meaning simply that the agent expects the same value that the variable was bound to before.

Fourth, we extend strands with events (predicates over terms) to formulate security goals in a protocol-independent way. For instance, we may use the event secret(A, B, Payload) to express that message Payload is regarded as a secret between protocol roles A and B. Then we can define (independent of the concrete protocol) a violation of secrecy as a state where the intruder has learned Payload but is neither A nor B. We do not give here more details on goals, because from a semantical point of view we just treat the events as if they were messages on a special channel to a "referee" who decides if the present state is an attack; the handling of these events is uniform for a wide class of goals [4] and only limited by the abilities of current verification tools. In textual representation, we will simply write event $(t)$ where $t$ is a term characterizing the event.

Fifth, we add checks of the form $s \doteq t$. The meaning is that the agent can only continue if the terms $s$ and $t$ are equal and aborts otherwise. Also, we have macros of the form $\mathcal{X}_{i}:=t$, which mean that we consider the same strand with all occurrences of $\mathcal{X}_{i}$ replaced by $t$. This is helpful for generating protocol implementations, because the result of a computation $t$ is stored in a variable $\mathcal{X}_{i}$ and does not need to be computed again.

A formal definition of operational strands can be given as a process (interacting with a given environment). In Appendix A, we define a semantics as state-transition systems similar to [15], where a state $(S ; K ; E)$ consists of a set $S$ of strands, a set $K$ of messages that the intruder currently knows and a set $E$ of events that have occurred. For instance, if $S$ contains the strand send $($ insec,$t)$.rest, where insec represents an insecure channel, then we can make the transition to a successor state where $t$ is added to $K$ and the send step is removed from the given strand. 


\section{SPS Semantics}

Above we described the SPS syntax for a fixed set of cryptographic operators (for which we later give a fixed set of algebraic equations). In this section, we give a semantics that is parametrized over an arbitrary set of operators and algebraic properties, inspired by $[24,14]$. One of the main contributions of our work is to give this general definition of a semantics for Alice-and-Bob style languages in a concise, mathematical way that is based on a few simple, general principles. The semantics is a function from SPS to (operational) strands; this function is in general not recursive as many of the underlying algebraic reasoning problems are not. The value of this general definition is its simplicity and uniformity: this is in fact the best mathematical argument why to define a concept in a particular way and not differently. In the next section, we then show that we can actually implement this semantics for the operators of SPS; in fact, we define a "low-level" semantics that is a computable function from SPS to strands (that is however so complicated that we give only an overview in this paper) and prove that it coincides with the general "high-level" semantics.

\subsection{Message model}

We define messages as algebraic terms and use the words message and term interchangeably. We distinguish two kinds of messages: the protocol messages that appear in an SPS specification and labels, which are the messages that the resulting strands are built from. It is necessary to make this distinction as the SPS specification reflects the ideal protocol run, while the semantics reflects the actual actions and checks that an honest agent performs in the run of the protocol. For this reason, we will also distinguish between two kinds of variables: protocol variables and label variables.

Definition 1. A message model is a four-tuple $(\Sigma, V, \mathcal{L}, \approx) . \Sigma$ is a countable set of function symbols, all denoted by lower-case letters, where: $\Sigma_{0} \subseteq \Sigma$ is a countable set of constants, $\Sigma_{p} \subseteq \Sigma$ is a finite set of public operators such as public-key encryption, and $\Sigma_{m} \subseteq \Sigma$ is a finite set of mappings (or private operators), disjoint from $\Sigma_{p}$, e.g., mapping from public to private keys. We assume a global public constant $\top \in \Sigma_{p} \cap \Sigma_{0}$. $V$ is a countable set of protocol variables. $\mathcal{L}=\left\{\mathcal{X}_{1}, \mathcal{X}_{2}, \mathcal{X}_{3} \ldots\right\}$ is a countable set of label variables disjoint from $\Sigma$ and $V . \approx$ is a congruence relation over ground terms over $\Sigma$ (i.e., terms without variables), which are denoted by $\mathcal{T}_{\Sigma}$. A term is thus a constant, a variable, or an application of a function (of $\Sigma$ ) on a term, and we write $\mathcal{T}_{\Sigma}(A)$ for the set of terms over signature $\Sigma$ and variables from set $A$.

As we define in a deduction relation below, the public operators in $\Sigma_{p}$ are those functions that every agent and the intruder can apply to messages they know, i.e., the cryptographic operators (including operators for decryption that do not occur in the SPS specification) and the non-cryptographic formats. In contrast, the mappings in $\Sigma_{m}$ are private, like shk in our example protocol. 
Table 1. Example of an equational theory $\approx$

\begin{tabular}{|l|l|}
\hline$(1) \operatorname{dscrypt}(k, \operatorname{scrypt}(k, m)) \approx m$ & $(2) \operatorname{vscrypt}(k, \operatorname{scrypt}(k, m)) \approx T$ \\
\hline$(3) \operatorname{dcrypt}(\operatorname{inv}(k), \operatorname{crypt}(k, m)) \approx m$ & $(4) \operatorname{vcrypt}(\operatorname{inv}(k), \operatorname{crypt}(k, m)) \approx \top$ \\
\hline$(5) \operatorname{open}(\operatorname{sign}(k, m)) \approx m$ & $(6) \operatorname{vsign}(k, \operatorname{sign}(\operatorname{inv}(k), m)) \approx \top$ \\
\hline For every $\mathrm{f} \in \Sigma_{f}$ with arity $\mathrm{n}$ and for every $i \in\{1, \ldots, n\}$ \\
$(7) \operatorname{get}_{i, \mathrm{f}}\left(\mathrm{f}\left(t_{1}, \ldots, t_{n}\right)\right) \approx t_{i}$ & $(8) \operatorname{verify} \mathrm{f}_{\mathrm{f}}\left(\mathrm{f}\left(t_{1}, \ldots, t_{n}\right)\right) \approx \top$ \\
\hline \hline$\left.(9) \exp \left(\exp \left(t_{1}, t_{2}\right), t_{3}\right)\right) \approx \exp \left(t_{1}, \operatorname{mult}\left(t_{2}, t_{3}\right)\right)$ & $(10) \operatorname{mult}\left(t_{1}, t_{2}\right) \approx \operatorname{mult}\left(t_{2}, t_{1}\right)$ \\
\hline$(11) \operatorname{mult}\left(t_{1}, \operatorname{mult}\left(t_{2}, t_{3}\right)\right) \approx \operatorname{mult}\left(\operatorname{mult}\left(t_{1}, t_{2}\right), t_{3}\right)$ \\
\hline
\end{tabular}

Example 1. As a concrete example of a message model that is representative for a large class of security protocols, let $\Sigma_{p}$ contain all operators of the equations in Table 1, where $\approx$ is the least congruence relation satisfying the equations. For instance, scrypt represents symmetric encryption, dscrypt is the corresponding decryption operator and vscrypt is a verifier: given a term $t$ and a key $k$, it tells us whether $t$ is a valid symmetric encryption with key $k$. This models the fact that most symmetric ciphers include measures to detect when the decryption fails (e.g., when it is actually not an encrypted message or the given key is not correct) and in concrete implementations this verification will be part of the call to dscrypt. We emphasize that our message model explicitly describes such fine details that most security protocol analysis tools silently assume; we could similarly define a set of primitives that do not allow verification and the semantics will accordingly define which verifications honest agents can and cannot do.

Similarly, the operators crypt, dcrypt and vcrypt formalize asymmetric encryption, and sign, open and vsign formalize digital signatures.

Let $\Sigma_{f} \subseteq \Sigma_{p}$ be a set of formats declared in an SPS specification. Then, for each format $f \in \Sigma_{f}$ of arity $n$, get ${ }_{i, \mathrm{f}} \in \Sigma_{p}$ is a an extraction function for the $i$-th field of the format (for all $1 \leq i \leq n$ ) and verify $\mathrm{f}_{\mathrm{f}} \in \Sigma_{p}$ is a verifier to check that a given message has format $f$.

Moreover, we have exp and mult for modular exponentiation and multiplication as needed in many Diffie-Hellman-based protocols. As is often done, we omit the modulus for ease of notation. $\Sigma_{p}$ also contains hash and mac representing hash and keyed hash functions, respectively (hash and mac do not appear in Table 1 since they have no algebraic properties).

Finally, a typical set of mappings could be: shk : Agent $\times$ Agent $\rightarrow$ SymmetricKey to denote a shared key of two agents, pk : Agent $\rightarrow$ PublicKey for the public key of an agent, and inv : PublicKey $\rightarrow$ PrivateKey for the private key corresponding to a given public key. Although pk is typically publicly available, it should not be a public operator as it does not correspond to a computation that honest agents or the intruder can perform (rather the initial distribution of keys should be specified in the knowledge section of SPS).

Definition 2. $A$ labeled message $t^{l}$ consists of a protocol message $t \in \mathcal{T}_{\Sigma}(V)$ and $a$ label $l \in \mathcal{T}_{\Sigma_{p}}(\mathcal{L})$. A knowledge is a substitution of the form $M=\left[\mathcal{X}_{1} \mapsto\right.$ $\left.t_{1}, \ldots, \mathcal{X}_{n} \mapsto t_{n}\right]$, where $\mathcal{X}_{i} \in \mathcal{L}$ and $t_{i} \in \mathcal{T}_{\Sigma}(V)$. We call the set $\left\{\mathcal{X}_{1}, \ldots, \mathcal{X}_{n}\right\}$ the domain of $M$ and write $|M|=n$ for the length of $M$. We may also refer to 
$M$ as a set of entries and write, e.g., $M \cup\left\{\mathcal{X}_{j} \mapsto t_{j}\right\}$ to add a new entry (where $\mathcal{X}_{j}$ is not in the domain of $M$ ).

Intuitively, the label variables represent memory locations of an honest agent. A label $l$ is composed from label variables and public operators, and reflects what actions an honest agent has performed on elements of its knowledge. A labeled message $t^{l}$ expresses that an honest agent performed the actions of $l$ to obtain what the SPS specification represents by term $t$. For instance, we represent the initial knowledge of $\mathrm{A}$ in Listing 1.1 by $\left[\mathcal{X}_{1} \mapsto \mathrm{A}, \mathcal{X}_{2} \mapsto \mathrm{B}, \mathcal{X}_{3} \mapsto \operatorname{shk}(\mathrm{A}, \mathrm{B})\right.$, $\left.\mathcal{X}_{4} \mapsto \mathrm{g}\right]$ to express that A stores her name and B's name in her memory locations $\mathcal{X}_{1}$ and $\mathcal{X}_{2}$, a key shared with $\mathrm{B}$ in $\mathcal{X}_{3}$, and the group g in $\mathcal{X}_{4}$.

\subsection{Message Derivation and Checking}

We now define how honest agents can derive terms from their knowledge. This is in the style of Dolev-Yao deduction relations, but extended to labeled messages to keep track of the operations that have been applied. The relation has the form $M \vdash t^{l}$ where $M$ is a knowledge and $t^{l}$ a labeled term. ${ }^{5}$

Definition 3. $\vdash$ is the least relation that satisfies the following rules:

$$
\overline{M \vdash t^{\mathcal{X}_{i}}} \begin{aligned}
& A x, \\
& {\left[\mathcal{X}_{i} \mapsto t\right] \in M}
\end{aligned} \quad \frac{M \vdash t^{l}}{M \vdash s^{m}} \stackrel{E q,}{s \approx t, l \approx m} \quad \frac{M \vdash t_{1}^{l_{1}} \ldots M \vdash t_{n}^{l_{n}}}{M \vdash f\left(t_{1}, \ldots, t_{n}\right)^{f\left(l_{1}, \ldots, l_{n}\right)}} \quad \begin{aligned}
& C m p, \\
& f \in \Sigma_{p}
\end{aligned}
$$

The rule $A x$ expresses that an agent can deduce any message that it has in its knowledge, $E q$ expresses that deduction is closed under equivalence in $\approx$ (on terms and their labels), and $C m p$ allows agents to apply any public operator to deducible terms. For example, under the theory of Table 1 , from $M=\left[\mathcal{X}_{1} \mapsto\right.$ $\left.\mathrm{k}, \mathcal{X}_{2} \mapsto \mathrm{X}, \mathcal{X}_{3} \mapsto \operatorname{scrypt}(\mathrm{k}, \exp (\mathrm{g}, \mathrm{Y}))\right]$ we can deduce $\exp (\exp (\mathrm{g}, \mathrm{X}), \mathrm{Y})$ as follows:

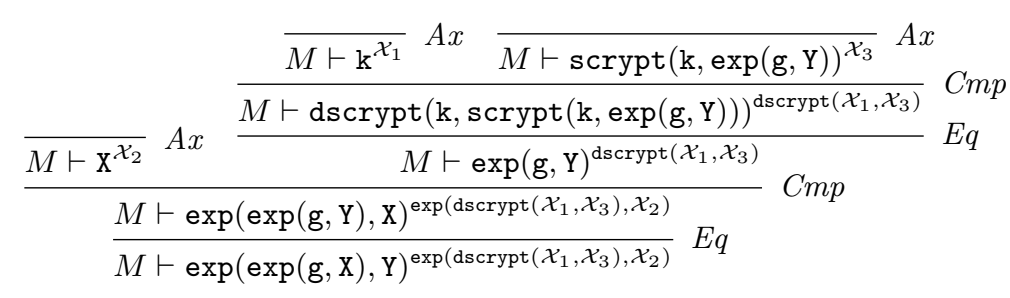

This tells us that the "recipe" to generate $\exp (\exp (\mathrm{g}, \mathrm{X}), \mathrm{Y})$ from $M$ is $\exp \left(\operatorname{dscrypt}\left(\mathcal{X}_{1}\right.\right.$, $\left.\mathcal{X}_{3}\right), \mathcal{X}_{2}$ ), which are the actual operations that the agent has to perform.

Based on the deduction relation, we define what checks an honest agent can perform on messages in his knowledge, e.g., whether a message can be successfully decrypted. We use the symbol $\doteq$ to talk about equations on terms of the

\footnotetext{
${ }^{5}$ One may employ an entirely different model for the intruder (e.g., a cryptographic one); using a Dolev-Yao style deduction for honest agents is simply the semantic decision that they perform only standard public operations (that would be part of a crypto API), but no operations that would amount to cryptographic attacks.
} 


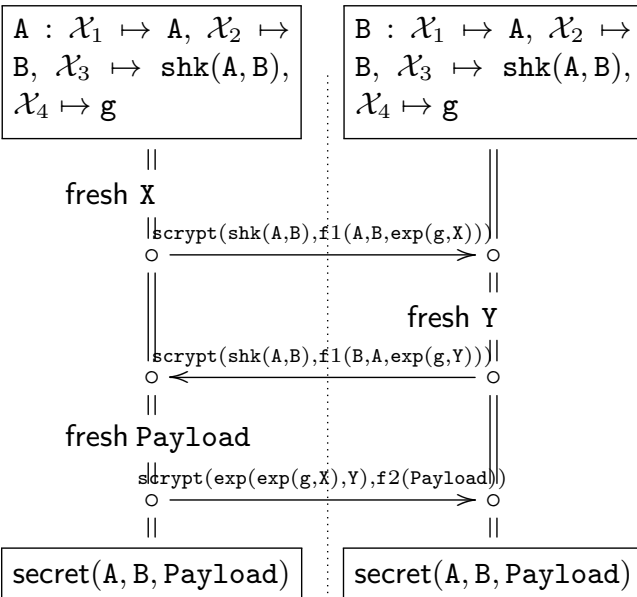

$\mathrm{B}, \mathcal{X}_{3} \mapsto \operatorname{shk}(\mathrm{A}, \mathrm{B})$

$\mathcal{X}_{4} \mapsto \mathrm{g}$

Fig. 1. (a) Example protocol

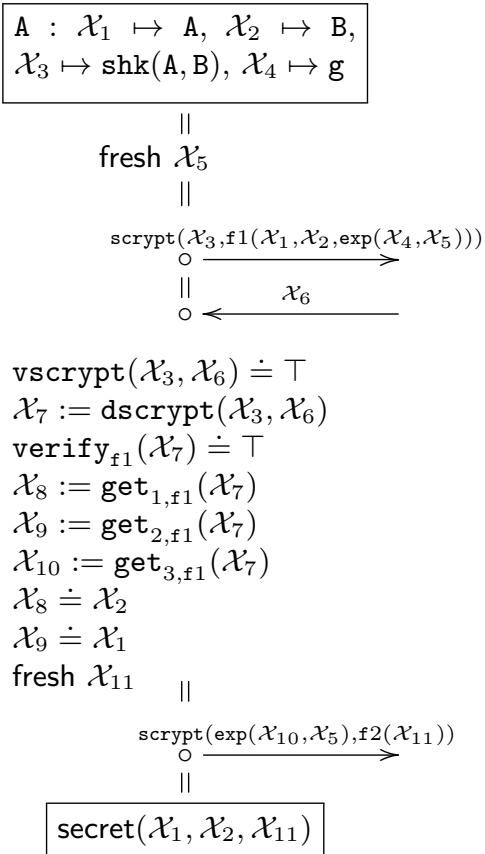

(b) Operational strand of A

labeling level and define $\doteq$-equations as follows: an interpretation $\mathcal{I}$ is a total mapping from $\mathcal{L}$ to $\mathcal{T}_{\Sigma}(V)$ that we extend to a function from $\mathcal{T}_{\Sigma}(V \cup \mathcal{L})$ to $\mathcal{T}_{\Sigma}(V)$ as expected; then we define $\mathcal{I}=s \doteq t$ iff $\mathcal{I}(s) \approx \mathcal{I}(t)$, and extend this to (finite or infinite) conjunctions of equations as expected. We define $\phi \models \psi$ iff $\mathcal{I} \models \phi$ implies $\mathcal{I} \models \psi$ for every interpretation $\mathcal{I}$; and $\phi \equiv \psi$ iff both $\phi \models \psi$ and $\psi \models \phi$.

Definition 4. We define a complete set of checks $\operatorname{ccs}(M)$ for a knowledge $M$ as follows: $\operatorname{ccs}(M)=\bigwedge\left\{l_{1} \doteq l_{2} \mid \exists m \in \mathcal{T}_{\Sigma}(V) . M \vdash m^{l_{1}} \wedge M \vdash m^{l_{2}}\right\}$.

$\operatorname{ccs}(M)$ yields an infinite conjunction of checks that an agent can perform on his knowledge. Intuitively, $M \vdash m^{l_{1}}$ and $M \vdash m^{l_{2}}$ expresses that, according to the SPS specification, computing $l_{1}$ and $l_{2}$ should yield the same result $m$, and the agent can thus check that they actually do. For instance, consider $M=$ $\left[\mathcal{X}_{1} \mapsto k, \mathcal{X}_{2} \mapsto \operatorname{hash}(m), \mathcal{X}_{3} \mapsto \operatorname{scrypt}(k, m)\right]$. Amongst others, $\operatorname{ccs}(M)$ then entails the checks $\phi=\operatorname{vscrypt}\left(\mathcal{X}_{1}, \mathcal{X}_{3}\right) \doteq \top \wedge \operatorname{hash}\left(\operatorname{dscrypt}\left(\mathcal{X}_{1}, \mathcal{X}_{3}\right)\right) \doteq \mathcal{X}_{2}$, i.e., the agent $A$ can verify that $\mathcal{X}_{3}$ is an encryption and that $\mathcal{X}_{2}$ is the hash of the content of the encrypted message $\mathcal{X}_{3}$. Note that there are many more equations (e.g., $\mathcal{X}_{1} \doteq \mathcal{X}_{1}$ ) and for every equation $s \doteq t$, we also have $h(s) \doteq h(t)$ for every unary public operator $h$. However, it holds that $\operatorname{ccs}(M) \equiv \phi$, i.e., $\operatorname{ccs}(M)$ is logically equivalent to $\phi$ and thus all other checks are redundant. 


\subsection{High-level Semantics}

We define the semantics of SPS specifications by translation to operational strands. Fig. 1(a) shows our example protocol in the style of message sequence charts. The first step towards an operational semantics is to split the protocol into different strands, one for each role, as indicated in Fig. 1(a) by the dotted line. We refer to the resulting strands as plain strands. Each plain strand shows how the protocol looks like from the point of view of that role in an ideal protocol run: what messages it is supposed to send and what messages it receives. The second step towards the operational semantics is to identify the precise set of actions, i.e., how messages are composed or decomposed, and what checks need to be performed on received messages. Fig. 1(b) shows how this operational description looks like for role A of the example (role B is very similar). In fact, one of the main problems that we solve in this work is to define the mapping from (a) to (b) in general and how to implement it for the example theory.

Now we put it all together to define the high-level semantics by the function $\llbracket \cdot \rrbracket_{H}$ (with initial case $\llbracket \cdot \rrbracket_{H_{0}}$ ), which translates from plain strands (the SPS representation) to operational strands (the actual actions of agents). In a nutshell, we use the labeled deduction $M \vdash t^{l}$ to define how an agent composes an outgoing message (or event), and we use the $c c s$ function whenever an agent receives a new message, formalizing the set of checks that the agent can perform at this point. Note that this is an infinite conjunction and we later show how to obtain an equivalent finite conjunction for the example theory.

Definition 5. $\llbracket \cdot \rrbracket_{H}$ translates from plain to operational strands as follows:

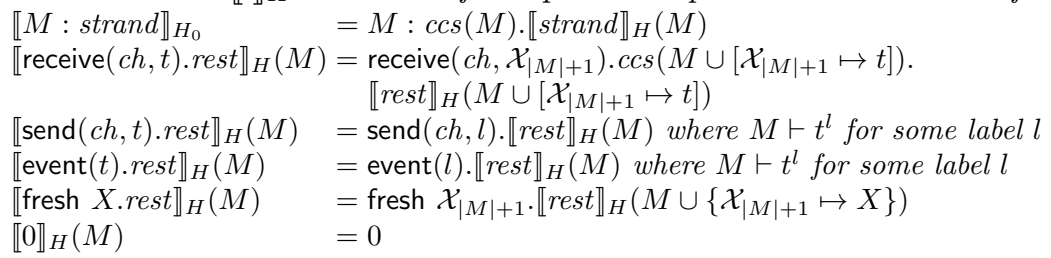

The first rule initializes the translation, by computing the checks that can be made on the initial knowledge of the strands. The second rule says that each received message is associated with a new label variable $\mathcal{X}_{|M|+1}$ in the agent's knowledge and afterwards we use ccs to generate all checks that the agent can perform on the augmented knowledge. The third rule is for sending the SPS protocol message $t$. (The following event rule is similar and thus we don't discuss it.) The relation $M \vdash t^{l}$ would thus hold for a label $l$, i.e., a concrete sequence of actions or "recipe", that would yield the term $t$ from the current knowledge $M$. The fifth rule translates the construct fresh $X$ : here we simply pick a new label variable $\mathcal{X}_{|M|+1}$ that will store the fresh value in the translated strand, and bind it in the knowledge to the protocol variable $X$. The final rule is straightforward.

Now consider an agent $A$ who has the knowledge $M=\left[\mathcal{X}_{1} \mapsto \mathrm{X}, \mathcal{X}_{2} \mapsto\right.$ $\exp (\mathrm{g}, \mathrm{Y})]$, i.e., the agent's own Diffie-Hellman secret and the Diffie-Hellman halfkey (supposedly) received from the other agent. For $A$, the recipe to generate the key $t=\exp (\exp (\mathrm{g}, \mathrm{Y}), \mathrm{X})$ is $l=\exp \left(\mathcal{X}_{2}, \mathcal{X}_{1}\right)$, but $A$ has (in our model) no 
means of checking that $\mathcal{X}_{2}$ has indeed been created by an exponentiation and would accept any "garbage" term for $\mathcal{X}_{2}$. The operational semantics thus tells us that the agent (no matter what $\mathcal{X}_{2}$ actually is) uses $\exp \left(\mathcal{X}_{2}, \mathcal{X}_{1}\right)$ to generate what shall be used as an encryption key for the payload message.

Given $M$ and $t$, there is in general not a unique $l$ such that $M \vdash t^{l}$. First, let us consider the case that there is no such $l$. In this case, the agent has no means (within the deduction relation) to obtain the term $t$ from its current knowledge. We thus say the protocol is non-executable and its semantics is undefined. This is an important sanity check on SPS specifications, namely that the user has not accidentally specified a protocol that does not work - which may easily happen in other formal languages like Applied $\pi$ and which can only be discovered by thorough checking of the model by a knowledgeable user.

Second, if there is a label $l$, then there will typically be infinitely many of them (trivially by performing redundant encryptions and decryptions). Our semantics does not prescribe which of the labels has to be taken (and the implementation below will take in some sense the simplest one). A key insight is that this does not make the semantics ambiguous: if $M \vdash t^{l_{1}}$ and $M \vdash t^{l_{2}}$ then $\operatorname{ccs}(M) \models l_{1} \doteq l_{2}$. Thus, since we always perform the checks on the knowledge after each received message, we know that the choice of labels does not make a difference.

As an example, observe that the operational strand we have given in Fig. 1(b) for our example protocol is correct according to this semantics (when resolving the $\mathcal{X}:=t$ macros): all outgoing messages have an appropriate label (for which $M \vdash t^{l}$ holds), and all checks $s \doteq t$ do indeed logically follow from $\operatorname{ccs}(M)$ for the respective $M$. In fact, we claim that the checks are logically equivalent to $\operatorname{ccs}(M)$, i.e., all other checks are redundant; it is part of the results of the next section to prove that and derive the given checks automatically.

We emphasize the succinctness of the definitions: Definitions 2-5 together fit on half a page and yet we define the semantics for an arbitrary set of cryptographic operators and algebraic properties. We believe that this is the best argument that the semantics of Alice-and-Bob notation should be defined this way - deriving from simple, general, uniform principles. However, this simple semantics cannot be directly used as a translator from Alice-and-Bob notation to formal models or implementations as it entails an infinite representation and several of the underlying algebraic problems are in fact not recursive in general.

Theorem 1 For a given strand $S$, the problem to compute a finite representation of $\llbracket S \rrbracket_{H}$, if it exists, is not recursive.

Proof Sketch. It is immediate that $\vdash$ is in general an undecidable relation (take an undecidable $\approx)$. Similarly, the relation $\{(M, s, t) \mid \operatorname{ccs}(M) \models s \doteq t\}$ is undecidable. It follows that for given a knowledge $M$, the problem to compute a finite conjunction $\phi$, such that $\phi \equiv \operatorname{ccs}(M)$, if one exists, is not recursive.

\subsection{Implementing the Semantics}

We can however give a more low-level, procedural semantics (that is actually computable) for a special theory and prove that it correctly implements the 
high-level semantics. More specifically, we now sketch how to actually compute the semantics for the example theory in Table 1 and where we additionally require that the SPS specification (and thus the plain strands) does not contain any destructors or verifiers.

Theorem 2 For our example theory in Table 1, for every strand $S$ in which no destructors or verifiers occur, $\llbracket S \rrbracket_{H}$ can be finitely represented and it is recursive.

Implementation/Constructive Proof. First we split the problem into a constructor and a destructor/verifier part (note they are not independent, e.g., in order to decrypt a message one may need to first compose a key). We also split the example theory into (i) equations $C$ that describe destructors and verifiers (the first 8 equations in the Table 1 ) and (ii) equations $F$ that just "rearrange" the term (the remaining equations). We then use equations $C$ as rewrite rules and apply them modulo $F$ (working on $F$-equivalence classes); the resulting rewrite relation $\rightarrow_{C / F}$ is convergent and we consider only normalized terms.

We define two functions, compose $_{M}(t)$ and $\operatorname{analyze}(M, \varphi)$. First, compose $_{M}(t)$ implements the "constructor" part of the $\vdash$ relation: find all labels $l$ such that $M \vdash t^{l}$ when using only constructors of $\Sigma_{p}$ (no destructors and verifiers) and using only equations from $F$. Note that the set of such labels $l$ is always finite. Second, analyze $(M, \varphi)$ starts with a knowledge $M$ and a set of checks $\varphi$ that have already been performed (so they do not need to be checked again). It computes a pair $\left(M^{\prime}, \varphi^{\prime}\right)$. Here, $M^{\prime}$ is analyzed, i.e., we have added to $M$ all subterms that can be obtained by applying destructors and normalizing the result; for this purpose, analyze calls the compose function to compose decryption keys when necessary. Also, for each decryption, the analysis will produce as part of $\varphi^{\prime}$ a new macro $\mathcal{X}_{i}:=l$ where $\mathcal{X}_{i}$ is the label variable in the augmented knowledge that holds the result of the decryption and $l$ is the recipe for obtaining it. Similarly, for each such decomposition step, we have a check from the respective verifier that is also added to $\varphi^{\prime}$. Further, analyze will check for every term whether there is a different way to of compose it (using again the compose function) and generate the equality. Finally, for all pairs of terms where the root operator is mult (and analogously for exp), we must check if the least common multiple can be generated from each of them. For instance, knowing $\left[\mathcal{X}_{1} \mapsto a b, \mathcal{X}_{2} \mapsto a c, \mathcal{X}_{3} \mapsto b, \mathcal{X}_{4} \mapsto c\right]$, we can derive the check $\mathcal{X}_{1} \mathcal{X}_{4} \doteq \mathcal{X}_{2} \mathcal{X}_{3}$.

We then show that for an analyzed knowledge, every derivable term can be derived using only compose and the checks resulting from analyze are equivalent to those of $c c s$. The full details of compose and analyze including the proofs can be found in Appendix B. Based on this, we obtain the following computable low-level semantics that mirrors the structure of the high-level semantics:

$$
\begin{aligned}
& \llbracket M: \operatorname{strand} \rrbracket_{L_{0}}(\emptyset, \top) \quad=M: \varphi \cdot \llbracket \operatorname{strand} \rrbracket_{L}\left(M^{\prime}, \varphi\right) \text { where }\left(M^{\prime}, \varphi\right)=\operatorname{analyze}(M, \top) \\
& \llbracket \text { receive }\left(\text { ch, t).rest } \rrbracket_{L}(M, \varphi)=\operatorname{receive}\left(c h, \mathcal{X}_{|M|+1}\right) \cdot \varphi^{\prime} \cdot \llbracket \text { rest } \rrbracket_{L}\left(M^{\prime},\left(\varphi \wedge \varphi^{\prime}\right)\right)\right. \\
& \text { where }\left(M^{\prime}, \varphi \wedge \varphi^{\prime}\right)=\text { analyze }\left(M \cup\left[\mathcal{X}_{|M|+1} \mapsto t\right], \varphi\right) \\
& \llbracket \operatorname{send}(c h, t) . r e s t \rrbracket_{L}(M, \varphi) \quad=\operatorname{send}(c h, l) \cdot \llbracket \text { rest } \rrbracket_{L}(M, \varphi) \text { where } l \in \text { compose }_{M}(t) \\
& \llbracket \operatorname{event}(t) \cdot \operatorname{rest} \rrbracket_{L}(M, \varphi) \quad=\operatorname{event}(l) . \llbracket \text { rest } \rrbracket_{L}(M, \varphi) \text { where } l \in \text { compose }_{M}(t) \\
& \llbracket \text { fresh X.rest } \rrbracket_{L}(M, \varphi) \quad=\text { fresh } \mathcal{X}_{|M|+1} \llbracket \llbracket \text { rest } \rrbracket_{L}\left(M \cup\left\{\mathcal{X}_{|M|+1} \mapsto X\right\}, \varphi\right) \\
& \llbracket 0 \rrbracket_{L}(M, \varphi) \\
& =0
\end{aligned}
$$




\section{Translations from Operational Strands}

We now come to the "last mile" of the translation: to translate operational strands into an actual implementation and into a formal model for automated verification. Fig. 5.2 shows this translation for the role A of our example in Fig. 1(b); the implementation language is JavaScript and the formal language is Applied $\pi .^{6}$ The close correspondence between these two translations allow us to argue that there is no discrepancy between formal model and implementation, if the functions symbols have the corresponding meaning - but that is indeed subtle. Comparing the translation with the input strand of Fig. 1(b), there are only two significant differences: all the explicit verifiers of the strands are removed and the implementation does not contain events; besides that, the translation is mainly adapting to the syntax of the target language. For this reason, we do not give here a formal definition of the translation functions (they can be found in Appendix C), but only discuss the necessary surrounding definitions and the subtleties in the meaning of the target languages.

\subsection{Experimental Results}

The translator has been implemented as part of the FutureID project and is available at [3]. In the project, we have considered several real world case studies such as the TLS handshake [16] as one of the most widely used protocols, the protocols EAC and PACE [19] that are used by the German eID card, and 30 smaller protocols. In particular, for our main case studies TLS, EAC and PACE, we did implement the precise message formats of the standards [18]. As part of FutureID, an execution environment has been defined that invokes the JavaScript code with suitable values for the parameters [17]. For the formal verification, we have used our case studies to check that ProVerif finds the known attacks in the small examples and verifies all other protocols. The entire test suite runs in less that 11 seconds on a $2.67 \mathrm{GHz}$ machine.

\subsection{JavaScript Translation}

Crypto API. We of course rely on the execution environment to have suitable implementations of the cryptographic primitives, e.g., the exp operator will in fact be mapped to elliptic curve cryptography. We assume that the call dscrypt $(k, m)$ will fail (aborting execution) if $m$ is not a message encrypted with key $k$. This is why we do not include verifier checks in this translation. For simplicity, we omitted the optional annotation of primitives with the precise algorithm and key length (that is only necessary when using different ones in the same protocol).

\footnotetext{
${ }^{6}$ One may argue that JavaScript is not suitable for implementing security protocols, but in fact, using systematic mechanisms such as our formats, we can produce robust implementations that do not suffer from type flaw attacks for instance. It is relatively easy to adapt to other languages like Java or the AVANTSSAR Platform [5], e.g., for using the tool OFMC, for which we have implemented a connector.
} 
The translator can only give a warning when the SPS specification is outside the fragment for which the soundness result holds.

Process Instantiation. We formulate the most general possible instantiation of the protocol: every role can be played by any agent, including the intruder, and we want to allow for any number of sessions of the protocol in parallel. It is not trivial to specify this manually, but the SPS compiler offers a systematic way to generate the instantiation. Recall that the initial knowledge of each role in the SPS specification can only contain variables of type Agent and long-term keys have to be specified using functions like shk. This allows us to instantiate the knowledge for any value of the role variables. For our example, we have the following specification (where the free name pub represents an insecure channel):

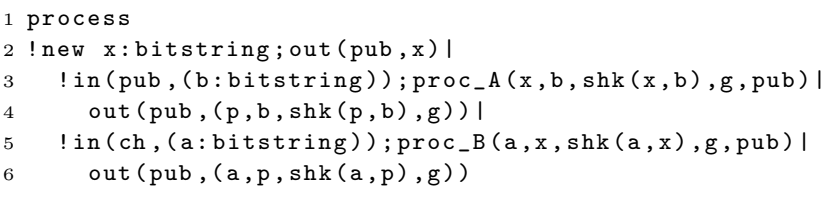

The first replication operator generates an unbounded number of honest agent names (in variable $\mathrm{x}$ ) that are broadcast on pub. Then we generate an unbounded number of instances of proc_A for each $\mathrm{x}$ and each name $\mathrm{b}$ that we receive from the public channel (thus, the intruder can choose who will play role $\mathrm{B}$ ). We also output on pub the initial knowledge that the intruder needs for playing role A under his real name $\mathrm{p}$. The last two lines are similar for role B.

\section{Conclusions and Related Work}

The formal definition of languages based on the Alice-and-Bob notation requires one to identify the concrete set of actions that honest agents have to perform, which is relevant both for a formal model for verification and for generating implementations. Previous works have proposed fairly involved deduction systems for this purpose and there is no (even informal) justification why these systems would be suitable definitions. Our high-level semantics $\llbracket \cdot \rrbracket_{H}$, inspired by $[24$, 14], gives a mathematically succinct and uniform definition of Alice-and-Bob notation following a few general principles, and at the same time it supports an arbitrary set of operators and algebraic properties. The succinctness and generality is, in our opinion, a strong argument for this semantics as a standard. As $\llbracket \cdot \rrbracket_{H}$ entails problems that are not recursively computable in general, we defined the low-level semantics $\llbracket \cdot \rrbracket_{L}$ for a particular theory and proved its correctness with respect to $\llbracket \cdot \rrbracket_{H}$. While $\llbracket \cdot \rrbracket_{L}$ is similar (and similarly involved) as previous definitions of semantics for the Alice-and-Bob notation $[22,23,13,12,20]$, we are the first to give a complete formal treatment of the key algebraic properties for destructors, verifies, exponentiation and multiplication.

With respect to other implementation generators like [31,28], our key improvements are as follows. First, we give a uniform way to generate both formal 
models and implementation from the operational strands, ensuring a one-toone correspondence between them. Second, replacing the abstract concatenation operator from formal models with formats allows us to generate code for any real-world structuring mechanism like XML formats or TLS-style messages. The only work that provides similar features is [7], which however starts at the $\pi$ calculus level, comparable to the output of our low-level semantics. In reference to works that consider the verification of the actual implementation source code like [8], we agree with [9] that the converse problem, i.e., turning formal models into code like in this paper, is harder. However, in the case of SPS this extra effort takes a large part of the burden off the user, i.e., SPS carries the task of formally verifiable implementations to a higher level of abstraction without suffering from flaws that are abstracted away in the formal model.

Finally, we point out a strong similarity between our notion of knowledge and the notion of frames in Applied $\pi$ calculus [2]. We allow ourselves minor deviations from the frame concept, in particular not using name restrictions; instead, constants are by default not public in our setting. This makes the treatment in this paper easier but does not fundamentally change the concept (or its expressive power). For what concerns existing decision results for frames, the deduction relation $\vdash$ has been studied, e.g., in [1]. It is known that deduction is decidable for convergent subterm theories (like our equations (1)-(8)) and that disjoint associate-commutative operators as in (9)-(11) can easily be combined with it. Many results consider the static equivalence of frames which is interesting for privacy properties, namely whether the intruder is able to distinguish two frames ("knowledges"). In the SPS semantics, we have a substantially different problem to solve: we have only one knowledge $M$ (and it is the knowledge of an honest agent) and we need to finitely characterize $\operatorname{ccs}(M)$, i.e., what checks the agent can make on $M$ to ensure that all received messages have the required shape. This indeed has some similar traits to static equivalence: also here, one has to check pairs of recipes (albeit with respect to two frames). Despite this similarity, the problems are so different that it seems not directly possible to reuse decision procedures for static equivalence for computing $\operatorname{ccs}(M)$. Moreover, our exp/mult theory is not yet supported in static equivalence results. A further investigation and generalization, namely with inverses for mult, is part of our ongoing research.

\section{References}

1. M. Abadi and V. Cortier. Deciding knowledge in security protocols under equational theories. Theor. Comput. Sci., 367(1-2):2-32, 2006.

2. M. Abadi and C. Fournet. Mobile values, new names, and secure communication. In C. Hankin and D. Schmidt, editors, POPL, pages 104-115. ACM, 2001.

3. O. Almousa. The SPS compiler. Available at http://www.imm.dtu.dk/ samo/.

4. O. Almousa, S. Mödersheim, P. Modesti, and L. Viganò. Typing and Compositionality for Security Protocols: a Generalization to the Geometric Fragment (Extended Version), 2015. Available at http://www.imm.dtu.dk/ samo/. 
5. A. Armando et al. The AVANTSSAR Platform for the Automated Validation of Trust and Security of Service-Oriented Architectures. In TACAS, 2012.

6. F. Baader and T. Nipkow. Term rewriting and all that. Cambridge University Press, 1998.

7. M. Backes, A. Busenius, and C. Hritcu. On the development and formalization of an extensible code generator for real life security protocols. In NFM, 2012.

8. K. Bhargavan, C. Fournet, R. Corin, and E. Zalinescu. Verified Cryptographic Implementations for TLS. ACM Trans. Inf. Syst. Secur., 15, 2012.

9. K. Bhargavan, C. Fournet, A. D. Gordon, and S. Tse. Verified interoperable implementations of security protocols. In CSF 19, pages 139-152. IEEE, 2006.

10. B. Blanchet. An efficient cryptographic protocol verifier based on Prolog rules. In CSF, pages 82-96. IEEE, 2001.

11. C. Bodei, M. Buchholtz, P. Degano, F. Nielson, and H. Riis Nielson. Static validation of security protocols. Journal of Computer Security, 13(3):347-390, 2005.

12. S. Briais and U. Nestmann. A formal semantics for protocol narrations. Theoretical Computer Science, 389(3):484-511, 2007.

13. C. Caleiro, L. Viganò, and D. Basin. On the semantics of Alice\&Bob specifications of security protocols. Theoretical Computer Science, 367(1):88-122, 2006.

14. Y. Chevalier and M. Rusinowitch. Compiling and securing cryptographic protocols. Information Processing Letters, 110(3):116-122, 2010.

15. C. Cremers and S. Mauw. Operational semantics of security protocols. In Scenarios: Models, Transformations and Tools, 2005.

16. T. Dierks and E. Rescorla. RFC 5246: The Transport Layer Security (TLS) Protocol, Version 1.2, 2008.

17. FutureID Project. Deliverable D42.6: Specification of execution environment, 2014. www.futureid.eu.

18. FutureID Project. Deliverable D42.8: APS Files for Selected Authentication Protocols, 2015. www.futureid.eu.

19. German Federal Office for Information Security (BSI). Advanced Security Mechanism for Machine Readable Travel Documents, 2008. Available at www.bsi.bund. de/EN/Publications/TechnicalGuidelines/TR03110/BSITR03110.

20. F. Jacquemard, M. Rusinowitch, and L. Vigneron. Compiling and verifying security protocols. In LPAR 2000, pages 535-554. Springer, 2000.

21. R. Küsters and T. Truderung. Using ProVerif to Analyze Protocols with DiffieHellman Exponentiation. In CSF, pages 157-171. IEEE, 2009.

22. G. Lowe. Casper: A compiler for the analysis of security protocols. In CSFW, pages 18-30. IEEE, 1997.

23. J. Millen. CAPSL: Common authentication protocol specification language. Technical report, Technical Report MP 97B48, The MITRE Corporation, 1997.

24. S. Mödersheim. Algebraic Properties in Alice and Bob Notation. In ARES'09, pages 433-440. IEEE, 2009.

25. S. Mödersheim. Diffie-Hellman without Difficulty. In FAST, pages 214-229, 2011.

26. S. Mödersheim and G. Katsoris. A sound abstraction of the parsing problem. In $C S F$, pages 259-273. IEEE, 2014.

27. S. Mödersheim and L. Viganò. Secure pseudonymous channels. ESORICS 2009, pages 337-354, 2009.

28. P. Modesti. Efficient Java Code Generation of Security Protocols Specified in AnB/AnBx. In STM, 2014.

29. M. Ryan and B. Smyth. Applied pi calculus. In Formal Models and Techniques for Analyzing Security Protocols. IOS Press, 2011. 
30. F. J. Thayer, J. C. Herzog, and J. D. Guttman. Strand spaces: Proving security protocols correct. Journal of Computer Security, 7(1):191-230, 1999.

31. B. Tobler and A. C. Hutchison. Generating network security protocol implementations from formal specifications. In Certification and Security in InterOrganizational E-Service. Springer, 2005. 


\section{A Operational Strands}

\section{A.1 The Syntax of Operational Strands}

The syntax of operational strands is a slight extension of the well-known strand spaces:

Strand $::=$ Knowledge: ( $\operatorname{send}($ Channel, Msg). | receive(Channel, MsG). $\mid$ event(MsG). $\mid$ MSG $\doteq$ MsG. $\mid$ VAR:=MsG. $\mid$ fresh VAR. $)^{*} 0$

Note that in plain strands, no equalities occur. The non-terminals CHANnEL, MsG, and VAR are as in the SPS syntax. KNOwLEDGE, typically denoted by $M$ in concrete strands, stands for a knowledge as defined in Definition 2, i.e., a substitution from label variables to protocol terms. We may omit this knowledge prefix of an operational strand when not relevant, as it is mainly used as an annotation in the semantics of SPS.

We define the free variables of an operational strand as follows:

$$
\begin{aligned}
f v(M: \text { rest }) & =f v(\text { rest }) \backslash \operatorname{dom}(M) \\
f v(\text { send }(\text { ch, } t) . \text { rest }) & =f v(\text { ch }) \cup f v(t) \cup f v(\text { rest }) \\
f v(\text { receive }(\text { ch, } t) . r e s t) & =(f v(\text { rest }) \backslash f v(t)) \cup f v(\text { ch }) \\
f v(\text { event }(t) . r e s t) & =f v(t) \cup f v(\text { rest }) \\
f v(s \doteq \text { t.rest }) & =f v(s) \cup f v(t) \cup f v(\text { rest }) \\
f v(x:=t . r e s t) & =(f v(\text { rest }) \backslash\{x\}) \cup f v(t) \\
f v(\text { fresh } x . r e s t) & =f v(\text { rest }) \backslash\{x\} \\
f v(0) & =\emptyset \\
f v(x) & =\{x\} \\
f v\left(f\left(t_{1}, \ldots, t_{n}\right)\right) & =f v\left(t_{1}\right) \cup \ldots \cup f v\left(t_{n}\right)
\end{aligned}
$$

We require that all operational strands are closed, i.e., all variables, before being "used", are bound by occurring in the knowledge, in a received message, or in a fresh step. Further, a bound variable cannot occur in a fresh step (e.g., fresh $x$.fresh $x .0$ is not allowed) or a macro (e.g., $x:=x$ cannot occur in a strand, since then $x$ is bound earlier, violating that it cannot be re-bound, or $x$ is a free variable of the strand). When a bound variable occurs in a receive step, it is not "re-bound", i.e., receive $(c h, x)$.receive $(c h, x)$.rest by the following semantics will be equivalent to receive $(c h, x)$.receive $(c h, y) . x \doteq y$.rest.

\section{A.2 The Semantics of Operational Strands}

Similar to [15], we define the semantics of operational strands as an infinitestate transition system, where a state $(S ; K ; E)$ consists of $(1)$ a set $S$ of closed strands, (i.e., every variable occurs first in a receive message, in a macro, or in a creation of a fresh value), (2) a set $K$ of messages (the intruder knowledge), and (3) a set $E$ of events that have occurred. This transition system is defined by an initial state and a transition relation. 
The initial state Recall that in an SPS specification, only variables of type agent may be used in a knowledge declaration; therefore the co-domain of the knowledge $M$ of each operational strand of the protocol will only contain such agent-typed variables. The first step in defining the semantics is to consider all possible instantiations of these agent variables with concrete agent names; and create infinitely many copies of these operational strands to model an unbounded number of sessions between any agents.

Let therefore $\mathfrak{S}=\left\{s_{1}, \cdots, s_{k}\right\}$ be the set of operational strands of a protocol, one for each role of the protocol. Let us further denote by $R_{i}$ the name of the role (i.e., a constant or variable of type agent) that is described by the operational strand $s_{i}, M_{i}$ be the knowledge of $s_{i}$ and $s t e p s_{i}$ be the steps of $s_{i}$, i.e., $s_{i}=M_{i}$ : steps $s_{i}$. Let $A g$ be a countably infinite set of constants of type Agent, including $\mathrm{p}$ denoting the intruder, and let $V_{A}$ be the set of all variables that occur in the $M_{i}$ (and are thus of type Agent in every SPS translation). Let $S u b s$ be the set of substitutions from $V_{A}$ to $A g$. Thus $S u b s$ represents all possible instantiations of the roles of the protocol with concrete agent names. When the SPS knowledge declarations contains some inequalities, such as $\mathrm{A} \neq \mathrm{p}$ or $\mathrm{A} \neq \mathrm{B}$, then this set Subs is accordingly restricted.

Even though a knowledge itself is a substitution (cf. Definition 2), we now define what it means to apply a substitution (from Subs) to it. Let $\sigma \in$ Subs and $M=\left[\mathcal{X}_{1} \mapsto t_{1}, \cdots, \mathcal{X}_{l} \mapsto t_{l}\right]$ be a knowledge. Then, we define $\sigma(M)=\left[\mathcal{X}_{1} \mapsto\right.$ $\left.\sigma\left(t_{1}\right), \cdots, \mathcal{X}_{l} \mapsto \sigma\left(t_{l}\right)\right]$. The initial state of the transition system is $\left(S_{0} ; K_{0} ; \emptyset\right)$ where:

$$
\begin{aligned}
& \left.S_{0}=\bigcup_{i=1}^{k}\left\{\sigma\left(M_{i}\right) \text { steps }_{i} \text {. finished }(n)\right) \mid \sigma \in \text { Subs, } \sigma\left(R_{i}\right) \neq \mathrm{p}, n \in \mathbb{N}\right\} \\
& K_{0}=\bigcup_{i=1}^{k}\left\{\sigma\left(u l\left(M_{i}\right)\right) \mid \sigma \in \operatorname{Subs}, \sigma\left(R_{i}\right)=\mathrm{p}\right\} \cup A g
\end{aligned}
$$

Here we use a new event finished $(n)$ (for each $n \in \mathbb{N}$ ) to create a countable number of unique operational strands for each instance $\sigma \in S u b s$. Note that we apply the instantiation $\sigma$ first to the knowledge of the role, and the so instantiated knowledge to the entire operational strand. For instance, for the trivial operational strand $\left[\mathcal{X}_{1} \mapsto \mathrm{A}, \mathcal{X}_{2} \mapsto B, \mathcal{X}_{3} \mapsto \operatorname{sk}(\mathrm{A}, \mathrm{B})\right]$ : fresh $\mathcal{X}_{4}$.send(insec, $\operatorname{scrypt}\left(\mathcal{X}_{3}, \mathcal{X}_{4}\right)$ and the instance $\sigma=[\mathrm{A} \mapsto \mathrm{a}, \mathrm{B} \mapsto \mathrm{p}]$, we get the countably many operational strands $\left[\mathcal{X}_{1} \mapsto a, \mathcal{X}_{2} \mapsto \mathrm{p}, \mathcal{X}_{3} \mapsto \operatorname{sk}(\mathrm{a}, \mathrm{p})\right]$ : fresh $\mathcal{X}_{4}$.send(insec, $\operatorname{scrypt}(\mathrm{sk}(\mathrm{a}, \mathrm{p})$, $\left.X_{4}\right)$ ). finished $(n) .0$ for each $n \in \mathbb{N}$. All remaining variables in the instantiated operational strands represent freshly created values and (parts of) received messages.

Note that here we have created only the instances for the honest agents (because of the side condition $\sigma\left(R_{i}\right) \neq \mathrm{p}$ ); this is so since the behavior of the honest agents is subsumed by the abilities of the intruder when given the appropriate knowledge of the role in all instances where he plays the role. ${ }^{7}$ With $K_{0}$ we

\footnotetext{
${ }^{7}$ In fact, we define here the semantics of operational strands using a standard DolevYao style intruder deduction relation; stronger models could be employed, we just require that the intruder can at least perform the actions that honest agents can, i.e., encryption and decryption with known keys and the like.
} 
therefore define the initial knowledge that the intruder needs to play in all roles under his real name. Here we model the intruder knowledge simply as a set of messages (rather than a substitution $M$ as for honest agents) as for the standard Dolev-Yao intruder deduction, we do not need labels (and we do not consider notions like behavorial equivalence here). Accordingly, let $\vdash^{\prime}$ denote the standard unlabeled intruder deduction on unlabeled messages, and the $u l$ function mapping from a knowledge to a set of terms by discarding the labels. Thus, $u l\left(\left[\mathcal{X}_{1} \mapsto t_{1}, \cdots, \mathcal{X}_{n} \mapsto t_{n}\right]\right)=\left\{t_{1}, \cdots, t_{n}\right\}$ and $u l(M) \vdash^{\prime} t$ iff $M \vdash t^{l}$ for some $l$.

The transition relation The transition relation $\Longrightarrow$ is defined as the least relation closed under the following rules:

$$
\begin{aligned}
& \text { T1 }(\{\text { send }(\text { insec }, t) . \text { rest }\} \cup S ; K ; E) \Longrightarrow(\{\text { rest }\} \cup S ; K \cup\{t\} ; E) \\
& \text { T2 }(\{\text { receive }(\text { insec }, t) . \text { rest }\} \cup S ; K ; E) \Longrightarrow(\{\sigma(\text { rest })\} \cup S ; K ; E) \\
& \text { for any substitution } \sigma \text { such that } K \vdash^{\prime} \sigma(t) \\
& \text { T3 }(\{\text { event }(t) . \text { rest }\} \cup S ; K ; E) \Longrightarrow(\{\text { rest }\} \cup S ; K ; E \cup\{\text { event }(t)\}) \\
& \text { T4 }(\{s \doteq \text { t.rest }\} \cup S ; K ; E) \Longrightarrow(\{\text { rest }\} \cup S ; K ; E) \quad \text { if } s \approx t \\
& \text { T5 }\left(\left\{\text { fresh } X_{i} . \text { rest }\right\} \cup S ; K ; E\right) \Longrightarrow(\{\sigma(\text { rest })\} \cup S ; K ; E) \\
& \text { where } \sigma=\left[\mathcal{X}_{i} \mapsto c\right] \text { and } c \text { is a fresh constant } \\
& \text { T6 }\left(\left\{\mathcal{X}_{i}:=t . r e s t\right\} \cup S ; K ; E\right) \Longrightarrow(\{\sigma(\text { rest })\} \cup S ; K ; E) \\
& \text { T7 }(\{0\} \cup S ; K ; E) \Longrightarrow(S ; K ; E)
\end{aligned}
$$

The rules $\mathrm{T} 1$ and $\mathrm{T} 2$ handle the sending and receiving over an insecure channel: we add every sent message $t$ to the intruder knowledge; for an agent who wants to receive a message of the form $t$ (note that $t$ may contain variables that are bound in this step), the intruder can use any instance $\sigma(t)$ that he can derive from his knowledge and we apply $\sigma$ to the rest of the strand, i.e., instantiating all variables that have been bound in this step. We have only discussed the standard case of insecure channels here, other kinds of channels can be defined as in the ideal channel model of [27].

Note that the following invariants holds over all transitions: the intruder knowledge is a set of ground terms, all strands are closed, and all terms that the intruder can derive and send are thus also ground.

The other rules should be self-explanatory. 


\section{B Message Composition and Decomposition}

In this section, we define the procedures for message composition and decomposition (compose and analyze respectively), but we first need some necessary definitions. First, we need to distinguish in the public operations between constructors and destructors.

Definition 6. Let $\Sigma_{d}=\{$ dscrypt, vscrypt, dcrypt, vcrypt, open, vsign, get, verify.\} be the destructors, where, abusing notation, we include get. and verify. for all formats. All other public operators $\Sigma_{c}=\Sigma_{p} \backslash \Sigma_{d}$ are called constructors.

Let us also denote by $\approx_{F}$ the least congruence relation that satisfies properties (9)-(11) in Table 1 that address modular exponentiation and multiplication. Since we have here no destructors for exp and mult, $\approx_{F}$ is a finite theory; i.e., for any term $t$, the equivalence class of $t$ under $\approx_{F}$ is finite (and moreover, unification is finitary, i.e., we can find finitely many most general unifiers for every pair of terms). We also define $\vdash_{C}$ as a restriction of $\vdash$ (Definition 3 ) where $\approx$ is replaced with $\approx_{F}$ and restricting $\Sigma_{p}$ to $\Sigma_{c}$. Thus, $\vdash_{C}$ is the "compositional" part of the $\vdash$ relation that allows only composing terms and application of $\approx_{F}$ (which never "decomposes" terms).

\section{B.1 Message Composition}

We now define the compositional part of message deduction, i.e., computing $\vdash_{C}$, realized by the function compose $_{M}(t)$ that computes all labels for generating the term $t$ from knowledge $M$ using only $\vdash_{C}$.

Definition 7. Let $M$ be a knowledge and $t \in \mathcal{T}_{\Sigma_{A}}(V)$.

$$
\begin{aligned}
\text { compose }_{M}(t)=\left\{\mathcal{X}_{i} \mid \exists t^{\prime} .\left[\mathcal{X}_{i}\right.\right. & \left.\left.\mapsto t^{\prime}\right] \in M \wedge t \approx_{F} t^{\prime}\right\} \cup \\
\left\{f\left(l_{1}, \ldots, l_{n}\right) \mid \exists\right. & t_{1}, \ldots, t_{n} . t \approx_{F} f\left(t_{1}, \ldots, t_{n}\right) \wedge f \in \Sigma_{c} \wedge \\
& \left.l_{1} \in \text { compose }_{M}\left(t_{1}\right) \wedge \ldots \wedge l_{n} \in \text { compose }_{M}\left(t_{n}\right)\right\} .
\end{aligned}
$$

The first part of compose $_{M}$ checks whether the term $t$ is directly contained in the knowledge modulo $\approx_{F}$, and returns corresponding label variables if so. The second part computes all ways to recursively compose $t$ from its direct subterms (modulo $\approx_{F}$ ). For instance, for $M=\left[\mathcal{X}_{1} \mapsto c, \mathcal{X}_{2} \mapsto \operatorname{hash}(c)\right]$ we have compose $_{M}(\operatorname{hash}(c))=\left\{\mathcal{X}_{2}, \operatorname{hash}\left(\mathcal{X}_{1}\right)\right\}$, and for $M=\left[\mathcal{X}_{1} \mapsto a \cdot b, \mathcal{X}_{2} \mapsto\right.$ $\left.c, \mathcal{X}_{3} \mapsto a \cdot c, \mathcal{X}_{4} \mapsto b\right]$ (writing $a \cdot b$ for $\operatorname{mult}(a, b)$ ), we have compose $_{M}(a \cdot b \cdot c)=$ $\left\{\mathcal{X}_{1} \cdot \mathcal{X}_{2}, \mathcal{X}_{3} \cdot \mathcal{X}_{4}\right\}$

The compose $_{M}$ function does not involve any decomposition steps or generate checks - for this we define an analysis procedure in the next subsection. The interface between the two procedures is the notion of an analyzed knowledge (in which every possible analysis step has already been done). We define this notion succinctly by requiring that every term that can be derived from $M$ using $\vdash$ can also be derived using $\vdash_{C}$, i.e., analysis steps do not yield any further messages: 
Definition 8. We say a knowledge $M$ is analyzed iff

$$
\left\{t \in \mathcal{T}_{\Sigma_{A}}(V) \mid \exists l . M \vdash t^{l}\right\}=\left\{t \in \mathcal{T}_{\Sigma_{A}}(V) \mid \exists l . M \vdash_{C} t^{l}\right\} .
$$

For an analyzed knowledge $M$, compose $_{M}$ is in fact correct:

Theorem 3 The compose $_{M}$ function terminates and is sound in the sense that $l \in$ compose $_{M}(t)$ implies $M \vdash t^{l}$. Moreover, if $M$ is analyzed and neither $M$ nor $t$ contain symbols from $\Sigma_{d}$, then compose ${ }_{M}$ is also complete in the sense that $M \vdash t^{l}$ implies $l^{\prime} \in$ compose $_{M}(t)$ for some label $l^{\prime}$ with $\operatorname{ccs}(M) \models l \doteq l^{\prime}$.

Proof. For termination, consider the tree of recursive calls that compose $_{M}(t)$ can invoke. The tree is finitely branching since $\approx_{F}$ is a finite theory (every term has a finite equivalence class). Suppose the tree has infinite depth, and let $t_{1}, t_{2}, t_{3}, \ldots$ be the terms in the recursive calls. Then there are terms $t_{1}^{\prime}, t_{2}^{\prime}, t_{3}^{\prime}, \ldots$ such that $t_{i} \approx_{F} t_{i}^{\prime} \sqsupset t_{i+1}$ for all $i \geq 1$. Then there are contexts $C_{1}[\cdot], C_{2}[\cdot], \ldots$ and $C_{i}[x] \neq x$ such that $t_{1} \approx_{F} C_{1}\left[t_{2}\right] \approx_{F} C_{1}\left[C_{2}\left[t_{3}\right]\right] \approx_{F} \ldots$ and thus $t_{1}$ has an infinite equivalence class modulo $\approx_{F}$, which is absurd, so the tree also has finite depth.

Soundness is immediate.

For completeness, consider $M \vdash t^{l}$, where $M$ is analyzed and $M$ and $t$ do not contain any symbols from $\Sigma_{d}$. Since $M$ is analyzed, we also have $M \vdash_{C} t^{l^{\prime}}$ for some $l^{\prime}$, and thus $\operatorname{ccs}(M) \models l \doteq l^{\prime}$. Due to $\vdash_{C}, l^{\prime}$ cannot contain any symbol from $\Sigma_{d}$ either (while $l$ can). Consider now the proof tree for $M \vdash_{C} t^{l^{\prime}}$ : leaf nodes are axioms and inner nodes are either composition steps with $f \in \Sigma_{c}$ or algebraic equivalences modulo $\approx_{F}$. It is straightforward to map them into corresponding steps of compose ${ }_{M}(t)$ to yield label $l^{\prime}$.

\section{B.2 Message Decomposition and Checks}

To compute an analyzed knowledge and the checks that one can perform on it, we define the procedure analyze that takes as input a pair $(M, \varphi)$ of a knowledge and a (finite) conjunction of equations and yields a saturated extension $(M \cup$ $\left.M^{\prime}, \varphi \wedge \varphi^{\prime}\right)$ of $(M, \varphi)$. The notion of saturated means that $M \cup M^{\prime}$ is analyzed and that $\varphi \wedge \varphi^{\prime}$ is equivalent to $\operatorname{ccs}\left(M \cup M^{\prime}\right)$. Note that this algorithm works incrementally, so when augmenting $M$ with a received message in the generation of operational strands, we do not need to start the analysis from scratch. Also, we assume that we never add redundant checks, i.e., ones that are already entailed by previous checks.

Table 2 summarizes the procedure analyze $(M, \varphi)$. The table is divided into two parts: the upper part represents the first phase of the algorithm, saturating $M$ with derivable subterms, whereas the lower part represents the second phase saturating $\varphi$ with additional equations.

Phase 1. Here we check for every entry in $M$ whether it can be analyzed, i.e., if it has one of the forms of column 1 (the head symbol being scrypt, crypt, sign, or a format) and that has not yet been marked as analyzed (initially no 


\begin{tabular}{|c|c|c|c|c|c|}
\hline 1. Term of the form & 2. & ondition & 3. Derive & 4. Check & 5. Recipe \\
\hline $\mathcal{X}_{i} \mapsto \operatorname{scrypt}(k, m)$ & $l \in$ & compose $_{M}(k)$ & $\mathcal{X}_{i^{\prime}} \mapsto m$ & $\operatorname{vscrypt}\left(l, \mathcal{X}_{i}\right) \doteq \top$ & $\| \mathcal{X}_{i^{\prime}}:=\operatorname{dscrypt}\left(l, \mathcal{X}_{i}\right)$ \\
\hline $\mathcal{X}_{i} \mapsto \operatorname{crypt}(k, m)$ & $l \in$ & compose $_{M}(\operatorname{inv}(k))$ & $\mathcal{X}_{i^{\prime}} \mapsto m$ & $\operatorname{vcrypt}\left(l, \mathcal{X}_{i}\right) \doteq \top$ & $\mathcal{X}_{i^{\prime}}:=\operatorname{dcrypt}\left(l, \mathcal{X}_{i}\right)$ \\
\hline $\mathcal{X}_{i} \mapsto \operatorname{sign}(\operatorname{inv}(k), m)$ & $l \in$ & $\operatorname{compose}_{M}(k)$ & $\mathcal{X}_{i^{\prime}} \mapsto m$ & $\operatorname{vsign}\left(l, \mathcal{X}_{i}\right) \doteq \top$ & $\mathcal{X}_{i^{\prime}}:=\operatorname{open}\left(l, \mathcal{X}_{i}\right)$ \\
\hline $\begin{array}{l}\mathcal{X}_{i} \mapsto \mathrm{f}\left(t_{1}, \ldots, t_{n}\right) \\
\text { for some } \mathrm{f} \in \Sigma_{f}\end{array}$ & $(\operatorname{trl}$ & & \begin{tabular}{|l}
$\mathcal{X}_{i_{1}^{\prime}} \mapsto t_{1}$ \\
$\mathcal{X}_{i_{n}^{\prime}} \mapsto t_{n}$ \\
\end{tabular} & verify $_{\mathrm{f}}\left(\mathcal{X}_{i}\right) \doteq \top$ & \begin{tabular}{|l}
$\mathcal{X}_{i_{1}^{\prime}}:=\operatorname{get}_{1, \mathrm{f}}\left(\mathcal{X}_{i}\right) \ldots$ \\
$\mathcal{X}_{i_{n}^{\prime}}:=\operatorname{get}_{n, \mathrm{f}}\left(\mathcal{X}_{i}\right)$ \\
\end{tabular} \\
\hline \multicolumn{6}{|c|}{\begin{tabular}{|c|c|c|c|} 
1. Term of the form $(i \neq j)$ & 2 . Condition (where $\left.\frac{t_{d}}{s_{d}}=\operatorname{shorten}\left(\frac{t_{1} \cdot \ldots \cdot t_{n}}{s_{1} \cdot \ldots \cdot s_{m}}\right)\right)$ & 3 . Check \\
\end{tabular}} \\
\hline \multicolumn{4}{|c|}{\begin{tabular}{|l|l|}
$\mathcal{X}_{i} \mapsto t$ & $\left\{l_{1}, \ldots, l_{n}\right\}=\operatorname{compose}_{M}(t)$ \\
\end{tabular}} & \multicolumn{2}{|c|}{$l_{1} \doteq l_{2} \doteq \ldots \doteq l_{n}$} \\
\hline \multicolumn{2}{|l|}{$\mathcal{X}_{i} \mapsto \operatorname{inv}(k)$} & \multicolumn{2}{|c|}{$l \in \operatorname{compose}_{M}(k)$} & & $\operatorname{vcrypt}\left(\mathcal{X}_{i}, \operatorname{crypt}(l, \top)\right) \doteq \top$ \\
\hline \multicolumn{2}{|l|}{$\begin{array}{l}\mathcal{X}_{i} \mapsto t_{1} \cdot \ldots \cdot t_{n} \\
\mathcal{X}_{j} \mapsto s_{1} \cdot \ldots \cdot s_{m}\end{array}$} & \multicolumn{2}{|c|}{$\begin{array}{l}l_{t_{d}} \in \operatorname{compose}_{M}\left(t_{d}\right) \\
l_{s_{d}} \in \operatorname{compose}_{\mathrm{M}}\left(s_{d}\right)\end{array}$} & & $l_{t_{d}} \cdot \mathcal{X}_{j} \doteq l_{s_{d}} \cdot \mathcal{X}_{i}$ \\
\hline \multicolumn{2}{|c|}{$\begin{aligned} & \mathcal{X}_{i} \mapsto \exp \left(A, t_{1} \cdot \ldots \cdot t_{n}\right) \\
& \mathcal{X}_{j} \mapsto \exp \left(A, s_{1} \cdot \ldots \cdot s_{m}\right)\end{aligned}$} & \multicolumn{2}{|l|}{$l_{t_{d}} \in$ compose $_{\mathrm{M}}\left(t_{d}\right)$} & & $\exp \left(\mathcal{X}_{j}, l_{t_{d}}\right) \doteq \exp \left(\mathcal{X}_{i}, l_{s_{d}}\right)$ \\
\hline \multicolumn{2}{|c|}{$\begin{aligned} \mathcal{X}_{i} & \mapsto \exp \left(A, t_{1} \cdot \ldots \cdot t_{n}\right) \\
\mathcal{X}_{j} & \mapsto s_{1} \cdot \ldots \cdot s_{m}\end{aligned}$} & $\begin{array}{l}l_{t_{d}} \in \operatorname{compose}_{\mathrm{M}}\left(t_{d}\right. \\
l_{A} \in \operatorname{compose}_{\mathrm{M}}(A \\
l_{s_{d}} \in \operatorname{compose}_{\mathrm{M}}(s\end{array}$ & & & $\exp \left(l_{A}, \mathcal{X}_{j} \cdot l_{t_{d}}\right) \doteq \exp \left(\mathcal{X}_{i}, l_{s_{d}}\right)$ \\
\hline
\end{tabular}

Table 2. Tabular overview of analyze $(M, \varphi)$

term is). We then check according to column 2 whether the necessary decryption key can be derived. For this, we use the compose $_{M}$ procedure yielding a label $l$ if the key is available; if there are several labels, we simply pick one. We then mark the entry $\mathcal{X}_{i} \mapsto \ldots$ as analyzed, choose a new label variable $\mathcal{X}_{i}^{\prime}$ and add the analyzed message to the knowledge $M$ according to column 3 . Further, we add the condition of column 4 and the recipe of column 5 to $\phi$. (We treat the recipe here like an equation for simplicity.) We repeat this until no more analysis step can be performed. (Note: whenever new terms are added to $M$, encrypted messages that have not been marked as analyzed need to be checked again.)

Phase 2. We now consider every entry of $M$ once and check for all alternative ways to generate it according to the first row in the lower part of the table. If we find more than one such label, we add the respective checks to $\phi$. The second row is to check if a private key fits to its corresponding public key if it is known. ${ }^{8}$

Next, we have rules for products and exponents (last three rows of the lower table). Here we consider any pair of entries in $M$ where the head symbol is exp or mult (according to the form of column 1), again writing · for multiplication. Here, we require a match such that none of the $s_{i}$ and $t_{i}$ is itself a product. We then consider the fraction $\left(t_{1} \cdot \ldots \cdot t_{n}\right) /\left(s_{1} \cdot \ldots \cdot s_{m}\right)$ and shorten it, i.e., removing common factors in enumerator and denominator. Let $t_{d} / s_{d}$ be remaining products after shortening. If all the $t_{i}$ or all the $s_{i}$ are shortened away (i.e., $t_{d}=1$

\footnotetext{
8 This check is actually quite academic, as the agent has either generated the key pair itself (and thus knows by construction that they form a key pair) or it has received it from a key server, e.g., in identity-based encryption (but then needs to trust that server anyway). However, without this check the correctness theorem and its proof would require a more complicated formulation.
} 
or $t s_{d}=1$ ) we do not apply this rule (as it is already covered by the first row, saving us from introducing 1 into the algebraic theory). We now try to compose the products $t_{d}$ and $s_{d}$ according to column 2. If there is at least one label for each (if there are several, again we pick one), then we add to $\phi$ the condition of column 3 .

Example 2. We compute analyze $(M, \top)$ for the knowledge $M=\left[\mathcal{X}_{1} \mapsto y\right.$, $\mathcal{X}_{2} \mapsto \operatorname{scrypt}(\exp (\exp (g, y), x), n), \mathcal{X}_{3} \mapsto \operatorname{scrypt}(k, \exp (g, x)), \mathcal{X}_{4} \mapsto k, \mathcal{X}_{5} \mapsto$ $\operatorname{hash}(n)]$.

For phase 1 , entries $\mathcal{X}_{1}, \mathcal{X}_{4}$, and $\mathcal{X}_{5}$ do not match any entry in the first column (they cannot possibly be decrypted). For $\mathcal{X}_{2}$, we have compose $_{M}(\exp (\exp (g, y), x))=$ $\emptyset$, i.e., the decryption key is not (yet) available. However, we can decrypt $\mathcal{X}_{3}$ since compose $_{M}(k)=\left\{\mathcal{X}_{4}\right\}$. We thus add $\mathcal{X}_{6} \mapsto \exp (g, x)$ to the knowledge, and to $\phi$ the check vscrypt $\left(\mathcal{X}_{4}, \mathcal{X}_{3}\right) \doteq \top$ and the recipe $\mathcal{X}_{6}:=\operatorname{dscrypt}\left(\mathcal{X}_{4}, X_{3}\right)$. We mark $\mathcal{X}_{3}$ as analyzed, and check again the unanalyzed $\mathcal{X}_{2}$. This time (for the updated $M)$ we have compose $_{M}(\exp (\exp (g, y), x))=\left\{\exp \left(\mathcal{X}_{6}, \mathcal{X}_{1}\right)\right\}$, and thus add $\mathcal{X}_{7} \mapsto n$ to the knowledge, and to $\phi$ the check vscrypt $\left(\exp \left(\mathcal{X}_{6}, \mathcal{X}_{1}\right), \mathcal{X}_{2}\right) \doteq \top$ and recipe $\mathcal{X}_{7}:=\operatorname{dscrypt}\left(\exp \left(\mathcal{X}_{6}, \mathcal{X}_{1}\right), \mathcal{X}_{2}\right)$. Since neither $\mathcal{X}_{6}$ and $\mathcal{X}_{7}$ can be further analyzed, phase 1 is finished. For phase 2 , we can of course re-construct the encryptions, e.g., $\operatorname{scrypt}\left(\mathcal{X}_{4}, \mathcal{X}_{6}\right) \doteq \mathcal{X}_{3}$ but that is already implied by the equation $\mathcal{X}_{6}:=\operatorname{dscrypt}\left(\mathcal{X}_{4}, \mathcal{X}_{3}\right)$ and we do not add redundant checks. The only new check is for $\mathcal{X}_{5}$, since compose ${ }_{M}(\operatorname{hash}(n))=\left\{\mathcal{X}_{5}\right.$, hash $\left.\left(\mathcal{X}_{7}\right)\right\}$ yields $\mathcal{X}_{5} \doteq \operatorname{hash}\left(\mathcal{X}_{7}\right)$.

As another example for equational reasoning, analyze $\left(\left[\mathcal{X}_{1} \mapsto a \cdot b \cdot c, \mathcal{X}_{2} \mapsto\right.\right.$ $\left.\left.a \cdot c \cdot d, \mathcal{X}_{3} \mapsto b, \mathcal{X}_{4} \mapsto d\right], \top\right)$ yields the check $\mathcal{X}_{1} \cdot \mathcal{X}_{4} \doteq \mathcal{X}_{2} \cdot \mathcal{X}_{3}$

Theorem 4 For a knowledge $M$ with no symbols in $\Sigma_{d}$ and a finite conjunction $\phi$ of equations, analyze $(M, \phi)$ terminates with a result $\left(M^{\prime}, \phi^{\prime}\right)$ such that $\{t \mid M \vdash$ $\left.t^{l}\right\}=\left\{t \mid M^{\prime} \vdash t^{l}\right\}$ (soundness), analyzed $\left(M^{\prime}\right)$ (completeness) and $\phi^{\prime} \equiv \operatorname{ccs}\left(M^{\prime}\right)$ (correctness of checks).

Proof. Soundness is immediate, as we merely add derivable messages to the knowledge.

Termination: The newly added terms of $M^{\prime}$ are always subterms of some term in $M$, so the $M^{\prime}$ component must eventually reach a fixed point. Adding new equations to $\phi^{\prime}$ is bounded by pairs of entries of $M^{\prime}$ and the finiteness of compose $_{M}$.

Completeness: We first show that $M^{\prime}$ is analyzed, i.e., we have to show that for any term $t \in \mathcal{T}_{\Sigma_{A}}(V)$ with $M^{\prime} \vdash t^{l}$, we also have $M^{\prime} \vdash_{C} t^{l^{\prime}}$ for some $l^{\prime}$ (i.e., using only constructors of $\Sigma_{c}$ and equivalence in $\approx_{F}$ ). For this, we consider the proof tree for $M^{\prime} \vdash t$. Intermediate nodes may well contain destructors, but we can exclude so-called garbage terms, namely terms that are not $\approx$-equivalent to any term in $\mathcal{T}_{\Sigma_{A}}(V)$. For instance, dscrypt $(c, c)$ is garbage (while $\operatorname{dscrypt}(k, \operatorname{scrypt}(k, m)) \approx m$ is not). Suppose the proof contains a node with a garbage term $s$, then there must be a construction in the proof to remove $s$ (since the final term must be in $\mathcal{T}_{\Sigma_{A}}(V)$ ), for instance constructing $\operatorname{dscrypt}(s, \operatorname{scrypt}(s, m)) \approx m$ eliminates garbage $s$, but in all such cases, 
all occurrences of $s$ must have been composed, so there exists a simpler proof without garbage.

We thus first show the following: for any $M^{\prime} \vdash t^{l}$ where $t$ is not garbage we have $M^{\prime} \vdash_{C} s^{k}$ for some $s \approx t$ and some label $k$. This is shown by induction over the proof tree of $M^{\prime} \vdash t^{l}$. For $A x$ and $E q$ the proof is immediate as well as for $C m p$ with $f \in \Sigma_{c}$. For $f \in \Sigma_{d}$, consider the term $t_{0}$ being analyzed. By induction $M^{\prime} \vdash_{C} s_{0}^{k_{0}}$ for some $s_{0} \approx t_{0}$, so this is (modulo $\approx_{F}$ ) either composed or an axiom. If it is composed, then the intruder decomposes a term he has composed himself and this proof can be simplified. If it is an axiom, then the intruder applies decomposition to a term in his knowledge, and analyze has already added the resulting term $t$ (modulo $\approx$ ) to $M^{\prime}$.

Note we have only proved that for $M^{\prime} \vdash t^{l}$ (where $t$ is not garbage) there is $M^{\prime} \vdash_{C} s^{k}$ for some $s \approx t$. We have to show that $M^{\prime} \vdash_{C} t^{l^{\prime}}$ for some $l^{\prime}$, but only for $t \in \mathcal{T}_{\Sigma_{A}}(V)$, i.e., without destructors. We claim that in this case we have $s \approx_{F} t$ (and thus follows $M^{\prime} \vdash_{C} t^{k}$ as $\vdash_{C}$ is closed under $\approx_{F}$ ). This claim follows from the fact that our destructor equations (1)-(8) can be read as rewrite rules (from left to right) that are convergent modulo $\approx_{F}$, and thus terms that do not contain constructors are in normal form modulo $\approx_{F}$. The idea for proving this convergence is that the rewriting rules have disjoint symbols from the equations in $\approx_{F}$ (so they cannot conflict) and we can prove convergence for the rewrite rules using the critical pair method, see e.g. [6].

Correctness of Checks: Now for $\phi^{\prime} \equiv \operatorname{ccs}(M)$. The soundness $(\operatorname{ccs}(M) \Longrightarrow$ $\phi)$ is obvious by checking that each step in analyze adds only sound equations. The completeness we prove again indirectly, i.e., suppose we have a term $t$ and two derivation proofs $M \vdash t^{l}$ and $M \vdash t^{l^{\prime}}$ such that $l \doteq l^{\prime}$ is not implied by $\phi^{\prime}$. Suppose in either of the derivation trees for $l$ and $l^{\prime}$ appears a composition step with a destructor. Suppose the message being decomposed is $t_{1}^{l_{1}}$ and the result of decomposing is $t_{0}^{l_{0}}$. Again assume that there are no decompositions in the subtrees. One possible case is the analysis of inv which is covered by the sixth case in analyze (Table 2). In all other cases, analyze $(M, \phi)$ must have added $t_{0}$ under some new label $X_{i}$ to $M^{\prime}$ and $\phi^{\prime}$ must entail $X_{i} \doteq l_{0}$ (and a constraint about verifiability of $l_{1}$ ). Let us thus replace the derivation $t_{0}^{l_{0}}$ with $t_{0}^{X_{i}}$ : this changes a subterm in labels $l$ and $l^{\prime}$, but for these changed labels still $l \doteq l^{\prime}$ does not follow from $\phi^{\prime}$. In this way we can step by step eliminate all analysis steps and thus have two trees without analysis for $t^{l}$ and $t^{l^{\prime}}$ such that $l \doteq l^{\prime}$ is not implied by $\phi^{\prime}$.

Now we consider the case that either of the two trees (say for $l^{\prime}$ ) is an application of only axiom and equality steps, thus $l^{\prime}=X_{i}$ for some variable $X_{i}$. Then $M^{\prime}$ contains $\left[X_{i} \mapsto t\right]$ for a term that can be composed in a different way using only constructors and $\approx_{F}$, i.e., $l \in$ compose $_{M}(t)$ and thus $\phi^{\prime}$ must contain $l \doteq l^{\prime}$, contradicting the assumption. Otherwise it must be two trees consisting of composition steps. We can exclude composition with any operator but exp or mult since otherwise we can simply go to one of the subterms. If it is exp or mult, then it has the form of adding factors to initially known exp or mult terms. Again we can exclude adding the same factor in both trees (since otherwise we 
can reduce again to a simpler case). The remaining case is however covered by our check rules for exp and mult, again showing that $l \approx l^{\prime}$ must by entailed by $\phi^{\prime}$. 


\section{Translating to Applied- $\pi$ Calculus}

Here, we present $\llbracket \cdot \rrbracket_{\pi}$ that translates an operational strand to an applied- $\pi$ calculus process. We use the syntax provided in [29]. Note that the semantics of operational strands is actually similar to a process calculus and this translation to it is mainly a matter of pretty-printing, yet some details that we explain shortly. We define $\llbracket \rrbracket_{\pi}$ as follows (+ denotes string concatenation):

$\llbracket M: \operatorname{strand} \rrbracket_{\pi} \quad=$ let $\operatorname{proc} \_+$own $(\operatorname{strand})+"("+\operatorname{par}(M)+")="+\llbracket$ strand $\rrbracket_{\pi}$ where: own(strand) is the name of the agent that owns the strand strand, and $\operatorname{par}(M)$ is a list of the process parameters derived from its initial knowledge $M$

$\llbracket \operatorname{send}($ ch,$l)$.rest $\rrbracket_{\pi}=$ "out(" + ch + ",$+1+"$ " $) ;+\llbracket$ rest $\rrbracket_{\pi}$

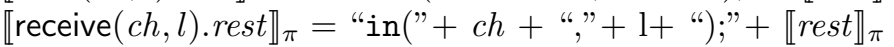

[fresh l.rest $\rrbracket_{\pi} \quad=$ "new" + l+ ":bitstring;" $+\llbracket$ rest $\rrbracket_{\pi}$

$\llbracket x:=$ t.rest $\rrbracket_{\pi} \quad=$ "let " $+\mathrm{x}+$ "=" $+\mathrm{t}+$ "in $"+\llbracket$ rest $\rrbracket_{\pi}$

$\llbracket t \doteq \top$.rest $\rrbracket_{\pi} \quad=\llbracket$ rest $\rrbracket_{\pi}$

$\llbracket s \doteq$ t.rest $\rrbracket_{\pi} \quad=$ "if(" $+\mathrm{s}+$ "=" $+\mathrm{t}$ " $)$ then " $+\llbracket$ rest $\rrbracket_{\pi}$

$\llbracket$ event $(t) \cdot$ rest $\rrbracket_{\pi} \quad=$ "event $(t) ; "+\llbracket$ rest $\rrbracket_{\pi}$

$\llbracket 0 \rrbracket_{\pi} \quad=$ " $0 . "$

The first rule declares the agent's process; by giving it a name and parametrize it over the initial knowledge of the agent. For example, Let $M^{A}: \operatorname{strand}^{A}$ be the strand shown in our example in Figure $1(\mathrm{~b})$, then own $\left(\right.$ strand $\left.^{A}\right)=\mathrm{A}$, and $\operatorname{par}\left(M^{A}\right)=\mathrm{x} 1, \mathrm{x} 2, \mathrm{x} 3, \mathrm{x} 4$ : bitstring, so the process will be proc_A as shown in the first line of the second column of Figure 5.2. The second and the third rules deal with the sending and receiving of messages over a channel $c h$. The forth rule deals with the creation of a fresh value, and the fifth rule covers the macro case of a strand and how it is translated in applied $\pi$ code. The sixth and the seventh rules deal with the checks. Note that in the case of a check that one of its sides is the true value $T$, we simply ignore such case since this check is implicitly performed by the next destruction step. For example, consider the translation of Figure $1(\mathrm{~b})$, we ignore vscrypt $\left(\mathcal{X}_{3}, \mathcal{X}_{6}\right) \doteq \top$ as it is followed by $\mathcal{X}_{7}:=\operatorname{dscrypt}\left(\mathcal{X}_{3}, \mathcal{X}_{6}\right)$, which according to the property (reduc forall m, k : bitstring; $\operatorname{dscrypt}(\mathrm{k}, \operatorname{scrypt}(\mathrm{k}, \mathrm{m}))=\mathrm{m}$.) will not be decrypted $\mathcal{X}_{6}$ unless it is a valid encrypted message and $\mathcal{X}_{3}$ is a valid encryption key. The eighth rule pretty-prints the event event $(t)$ in the process and the last rule ends the strand. 